\title{
O metamorfismo tecnológico em sala de aula: entrevista com o professor Alckmar Luiz dos Santos
}

\author{
Alckmar Luiz dos Santos ${ }^{\mathrm{i}}$ \\ Entrevistadoras: \\ Gabriela Ribeiro Nunes ${ }^{\text {ii }}$ \\ Marcela Santos Brigida ${ }^{\text {iii }}$
}

Um dos entrevistados desta nova edição da revista Palimpsesto é o professor da Universidade Federal de Santa Catarina, Alckmar Luiz dos Santos. Graduado em Engenharia Eletrônica pela Universidade Estadual de Campinas (1983), é mestre em Teoria e História Literária pela mesma instituição e doutor em Estudos Literários pela Université Paris VII. Desde 1995, atua como coordenador do Núcleo de Pesquisas em Informática, Literatura e Linguística (NUPILL), onde desenvolve pesquisas sobre os textos literários em meio digital, explorando os recursos que a informática e as tecnologias de informação e comunicação trazem para a pesquisa em Literatura e em Linguística. É também poeta, romancista, ensaísta e criador digital. Dentre sua vasta produção literária, destacamos o livro de poemas Rios Imprestáveis (2001), vencedor do Prêmio Redescoberta da Literatura Brasileira da Revista Cult e o romance Ao que minha vida veio... (2011), ganhador do Prêmio de Romance Salim Miguel.

\footnotetext{
${ }^{i}$ Possui graduação em Engenharia Eletrônica pela Universidade Estadual de Campinas (1983), mestrado em Teoria e História Literária pela Universidade Estadual de Campinas (1989) e doutorado em Estudos Literários pela Université Paris VII (1993). Desde 1994, é professor de Literatura Brasileira da Universidade Federal de Santa Catarina (UFSC) e, a partir de 1995, coordenador do Núcleo de Pesquisas em Informática, Literatura e Linguística (NUPILL, núcleo de pesquisa de excelência do CNPq, financiado pelo edital PRONEX entre 2008 e 2016). Foi pesquisador convidado na Université Paris 3 Sorbonne Nouvelle (2000-2001) e na Universidad Complutense de Madrid (2009-2010). Tem experiência na área de Letras, com ênfase em Literatura Brasileira e Teoria Literária, atuando principalmente com teoria do texto, literatura e filosofia, hipertexto e texto digital, poesia.

Site do NUPILL: http://nupill.ufsc.br/

ii Doutoranda em Teoria da Literatura/Literatura Comparada pela Universidade do Estado do Rio de Janeiro (UERJ). https://orcid.org/0000-0002-6593-0351|j15c17g11@hotmail.com

iii Doutoranda em Estudos Literários na Universidade do Estado do Rio de Janeiro (UERJ). Bolsista CNPq. https://orcid.org/0000-0002-0951-1603 | marcela.brigida@uerj.br
} 
O proeminente currículo do professor Alckmar, embora traduza sua qualidade como pesquisador, docente e escritor, não manifesta sua gentileza e humildade. Ficamos imensamente agradecidas pela maneira solícita com que ele aceitou o nosso convite para ser o entrevistado deste número cujo tema é "Ensino de literatura em contextos digitais: diálogos e conexões”. Foi uma experiência gratificante e enriquecedora aprender através das reflexões realizadas no que diz respeito ao uso de tecnologias digitais em sala de aula e das indicações de ferramentas que auxiliam os professores no ensino de literatura. Sua modéstia em citar seus amigos e colegas de caminhada, além do didatismo com que passou suas ideias nos inspiram e convidam a atualizarmos nossas práticas docentes, assim como faz o professor Alckmar. Tenham todos e todas uma boa leitura!

\section{PALIMPSESTO}

1) Em tempos de pandemia, mais do que nunca, o ensino/aprendizagem em contexto digital tem sido uma realidade para professores e alunos. Como pesquisador da área, quais são as vantagens e desvantagens que tal meio traz no que tange ao ensino de Literatura?

\section{ALCKMAR LUIZ DOS SANTOS}

Como professor, tive algumas experiências com ensino a distância que não foram sempre boas. Primeiramente, porque havia alguma pressão do MEC para transformar o ensino a distância em números para serem propagandeados, sem que predominasse a preocupação com a qualidade. Quando preparávamos a primeira graduação em Letras a distância, pela UAB, Universidade Aberta do Brasil, um representante do MEC veio conversar para expor as diretrizes. Ele dizia que os alunos disporiam de cinco a, no máximo, dez horas por semana de estudo. Era com isso que tínhamos que contar. Ora, eles iriam receber um diploma igual ao dos outros que dedicavam entre 30 e 40 horas por semana, o que pressupõe que deveriam todos ter uma formação equivalente. Evidentemente, a conta não fecha.

De outro lado, havia discentes que achavam que ensino a distância era sinônimo de facilidade, exigindo um esforço menor para alcançar a formação. Todavia, o trabalho é muito maior, pois o aluno precisa ter muito mais disciplina, cuidado, atenção e 
consciência do que pode e do que vai fazer. É preciso também que ele extraia o máximo possível das plataformas, do material à disposição, do professor, dos tutores. Sendo assim, há problemas que devem ser pensados de todos os lados: das instituições que organizam esse ensino, dos professores e dos alunos. Com a pandemia isso se acelerou muito, mas, em princípio, não vejo essa entrada no ensino remoto necessária ou exclusivamente como algo ruim. Havia muito receio de colegas meus, professores, de usarem esse tipo de recurso, quando, na verdade, não deve haver receio. Nós temos que usar e melhorar cada vez mais. Se há problemas, só vamos conseguir resolvê-los botando a mão na massa. A pandemia, portanto, ajudou nesse sentido, já que agora todo mundo teve que entrar na dança.

\section{PALIMPSESTO}

2) O senhor é coordenador do Núcleo de Pesquisas em Informática, Literatura e Linguística (NUPILL), desde 1995. De onde surgiu o seu interesse por pensar na relação Informática/Literatura e qual é a importância de se compreender essa dinâmica para um professor da disciplina? Isso influenciou na sua prática docente em que sentido?

\section{ALCKMAR LUIZ DOS SANTOS}

Quando entrei na Universidade Federal de Santa Catarina, tinha acabado recentemente o doutorado. Trabalhei com epistemologia, crítica literária, filosofia, poesia, literatura. Entretanto, a minha primeira graduação foi em Engenharia Eletrônica. Não posso dizer hoje que sou engenheiro eletrônico, nem de longe. Essas coisas ficaram em um passado remoto e indistinguível. Atualmente, me lembro de pouca coisa, mas a base do raciocínio ficou, assim como o interesse e certa facilidade em usar as ferramentas digitais.

Desde o final do meu mestrado na UNICAMP, já usava editor de texto para a elaboração da dissertação e depois, no doutorado, usei muito o computador. Utilizei também uma base de dados para catalogar as leituras realizadas, além de buscar os conteúdos dessas leituras, uma vez que muito dos fichamentos que fiz foram digitalizados. Consequentemente, eu já tinha uma familiaridade com a tecnologia. Além 
disso, desde os 8 anos de idade, veio a relação criativa com a literatura, já que com essa idade me meti a ser poeta. Toda essa trajetória fez com que, posteriormente, começasse a me perguntar o porquê de eu não usar a ferramenta digital para a criação literária visto que a utilizava para a produção intelectual acadêmica. Foi dessa forma que o primeiro clarão veio. Ao lado disso também surgiu o lado pragmático de testar para ver no que daria. Comecei a fazer experimentos de criação com meu amigo Gilbertto Prado, professor da USP.

Refletindo sobre o uso que fiz das ferramentas digitais para a produção da tese, de ensaios e de artigos, cheguei à conclusão de que seria interessante ter acesso a documentos, recursos e informações do âmbito literário de modo rápido, utilizando a Internet. Dessa forma, surgiu a ideia de fazer uma biblioteca digital, começando pela obra de Machado de Assis. Esse foi o objetivo inicial do NUPILL, em 1995. Logo outros objetivos se somaram a esse, como a ideia de fazer um banco de dados de história literária associado à biblioteca digital. Essa ideia não foi minha. Peguei isso do grupo Hubert de Phalèse, da Paris 3, onde fiz meu pós-doutorado, entre 2000-2001. Até hoje eles têm disponível o banco de dados da literatura francesa. No nosso caso, fizemos da literatura brasileira.

Tudo isso funcionou muito em minha prática docente. Só lamento que muitos colegas não usem também. A facilidade que temos hoje de acessar e cruzar dados e informações é enorme, e o banco de dados da biblioteca digital já é bastante grande e continua aumentando. Abstraindo o fato de que sou um dos que ajudaram a construir tudo isso, a biblioteca digital e o banco de dados são valiosíssimos, para qualquer professor de literatura. Temos por volta de 80 mil obras cadastradas e em torno de 6.500 obras digitalizadas. Temos mais de 22 mil autores de literatura de língua portuguesa Brasil, Portugal e África. África, infelizmente, ainda muito pouco; Portugal começa a crescer agora; do Brasil vem o grosso dos nossos dados. Isso ajuda muito, pois possibilita o acesso às obras, às informações sobre os autores, aos pseudônimos, aos subtítulos, aos gêneros. Dando aula, constantemente digo: "Vamos verificar isso na biblioteca digital, vamos ver isso no banco de dados". Se alguém quiser saber, por exemplo, quantos livros de teatro foram publicados entre 1850-1880 por mulheres em Portugal, é um dado facilmente encontrado em nosso banco de dados. Sem contar que, aos poucos, graças ao trabalho com um amigo e pesquisador da computação da UFSC, 
Roberto Willrich, desenvolvemos ferramentas como o DLNotes, para anotações em arquivos literários e arquivos $H T M L$. No nosso caso, usamos para obra literária. Essa ferramenta de anotações é utilíssima em sala de aula, uma vez que dinamiza e enriquece a leitura do aluno. Depois que começamos a utilizar o DLNotes, em 2010, as aulas, o diálogo com os alunos e o que eles trazem como informação e entendimento da obra melhoraram muito.

Há também outro amigo, Adiel Mitmann (e também pesquisador do NuPILL), que no doutorado desenvolveu uma ferramenta chamada Aoidos, responsável por fazer a escansão automática de versos, independentemente do tamanho. Faz a escansão tanto de uma quadrinha, quatro versos, em milésimos de segundo, quanto de um corpus de 1 milhão de versos, quantificando todos os fenômenos de acomodação silábica, como sinalefa, diérese, sinérese, ectlipse, suarabácti etc. Além disso, é capaz de mapear e quantificar os ritmos e os metros usados pelos poetas, sendo, portanto, uma ferramenta valiosíssima para quem trabalha com versos. A questão, agora, é que nossos colegas professores se disponham a dar o passo de mergulhar na tecnologia digital. Se usamos tanto celular e smartphones, lançar mão dessas ferramentas digitais não é um esforço assim tão grande.

\section{PALIMPSESTO}

3) Quais mudanças mais significativas o senhor, como coordenador do NUPILL há mais de 25 anos, enxergou no trato do texto literário e no ensino/aprendizagem de Literatura em contexto digital?

\section{ALCKMAR LUIZ DOS SANTOS}

Em relação ao ensino/aprendizagem é basicamente o que respondi na pergunta anterior. Tenho visto os colegas cada vez mais interessados, sobretudo os mais jovens que estão chegando nas universidades. Eles já têm uma familiaridade com tudo isso, então a facilidade deles de usar essas ferramentas e estratégias é muito maior e isso faz muita diferença.

Já no trato com o texto literário, nos anos 90 e 2000, houve uma grande euforia com isso. Nos Estados Unidos, há nomes como o do Michael Joyce e na França há o 
meu amigo, Philippe Bootz, trabalhando com essa questão. Muitas pessoas, inclusive eu, também estávamos buscando formas novas. No meu caso, sempre fui atrás de fazer com que elementos novos dialoguem com antigos. Nunca acreditei na história de começar uma criação ou um pensamento a partir do zero. Isso me faz lembrar daquela bela obra literária que é o Eclesiastes (1:9): "Nada de novo sob o sol". O novo, muitas vezes, é uma nova forma de usar o que veio de antes e que você aprendeu com os outros.

Houve, então, uma busca muito intensa de um lado e de outro, com resultados desiguais, mas diria que sempre interessantes. Sem querer ser indulgente, é uma bela tentativa que até hoje se faz de buscar o diálogo com o novo e com esses novos meios. São novas dinâmicas, novos espaços e uma nova maneira de pensar as questões todas do literário. De fato, o que a gente viu foi uma tentativa, às vezes disfarçada, às vezes explícita, de conciliar três eixos. Um eixo é a literatura verbal, o outro é o semiótico, ou seja, a imagem e o som que ganham um novo relevo nessas criações, e último é o computacional. Os três eixos (verbal, semiótico e computacional) aparecem sempre. Os autores e criadores que acho mais interessantes tinham consciência disso e tentavam fazer esses três eixos dialogarem de modo interessante e rico, cada um com seu estilo. Outros faziam isso sem muita consciência, então víamos muitos casos de poesia eletrônica ou poesia digital que, na verdade, era o velho verso feito para a tradição impressa, simplesmente colocado na tela de um computador; assim como muita gente falava de literatura digital, literatura informatizada, literatura computacional, literatura eletrônica, quando era simplesmente a literatura feita em papel, digitalizada e posta para ser lida na tela. Pouca gente fazia o que seria uma verdadeira literatura digital ou computacional ou eletrônica. No meu caso, prefiro chamar sempre de literaturas digitais - aquelas que não têm como ser impressa, porque o papel da interação computacional é muito grande. A interação entre as várias semióticas com o verbal é muito intensa também.

Depois dessa euforia de aprendizado, o que eu vejo hoje é que há algumas vertentes já se consolidando. Uma delas é a geração automática, que vem desde os anos 1950; ainda há muita gente trabalhando com isso de forma interessante. Outra vertente que se fortaleceu muito nos últimos anos é a do diálogo com os vídeojogos. Inclusive, um teórico da literatura, Espen Aarseth, disse que deixou de ser crítico literário para 
virar um estudioso dos jogos. Há quem trabalhe mais com o lado do visual. No nosso caso, por exemplo, a última criação que fizemos e estamos acabando agora se chama $e$ Imigrações. Trata-se de conciliar a criação verbal com o dado semiótico de som e imagem e com a interatividade computacional, trazendo o lado visual da história em quadrinho. Dessa forma, existe uma gama muito heterogênea de elementos possíveis de serem reunidos para dar-lhes um sentido. O grande problema é realmente esse sentido mais unívoco, porque muitas vezes é fácil juntar elementos díspares e heterogêneos e, no fim, se deparar com um Frankenstein que não se mexe, é morto e não anda - partes incomponíveis associadas de modo artificial.

\section{PALIMPSESTO}

4) Para o senhor, como o contexto online influencia no modo de se produzir e consumir literatura? Há uma reconfiguração no papel do autor, do leitor e do próprio texto literário?

\section{ALCKMAR LUIZ DOS SANTOS}

Claro que sim. Para começar, em termos de teoria literária, deve-se fazer uma diferença muito clara entre autor e escritor. O escritor é a pessoa de carne, osso, sangue, vida, CPF, RG, família, profissão, peso, altura, cor dos olhos, cabelo. Já o autor é um elemento textual que vai aparecer na hora em que há uma leitura - entendida do modo mais amplo possível: desde leitura de palavras em uma folha de papel impresso até a leitura de uma obra digital interativa. O autor, então, é um elemento textual que faz parte do processo de leitura.

Em relação às novas configurações, há, por exemplo, escrita e criação coletivas. Quando é feita uma criação como a que me referi na pergunta anterior, e-Imigrações, não posso dizer que eu sou o criador. Os criadores somos o Vinícius Rutes, o Rafael Duarte e eu. Nós três estamos trabalhando com isso, cada um com a sua contribuição. Evidentemente, pendo mais para o lado da criação verbal e das interações, que é algo com que venho mexendo há muito tempo. O Rafael pende mais para o lado da visualidade. A tese dele foi sobre história em quadrinhos e poesia visual. Já o Vinícius tem colaborado muito com a programação, que eu e o Rafael não dominamos, sem 
deixar de lado uma consciência do verbal. Cada um fica responsável por uma parte específica, mas participa das outras também. Dessa forma, há uma criação coletiva. Entretanto, essas criações diferentes de autorias distintas não são específicas agora da cultura digital. Um exemplo são os centões - tipo de composição poética da Idade Média, em que poetas medievais pegavam versos da Eneida, do Virgílio, combinavam e faziam um poema. Essa tradição dos centões se perpetuou. No século XVIII, nas Academias do Brasil, um poeta chamado Antônio de Oliveira fez um poema chamado: "Soneto encontrado no poema do príncipe dos poetas espanhóis". O príncipe dos poetas espanhóis é Camões. Na época, a Espanha era a Península Ibérica. Espanhol entendia-se como hoje entendemos ibérico. Antônio de Oliveira pegou 14 versos ao longo d'Os Lusíadas e fez um soneto em homenagem à Academia de que fazia parte. O poema é sintaticamente coerente, semanticamente correto, é um soneto, com versos decassílabos heroicos, o esquema de rimas é o mais tradicional, mas... quem é o criador desse soneto? Camões? Os versos são dele, mas Camões nunca na vida imaginou que haveria esse soneto dentro d'Os Lusíadas. Então o criador é o Antônio de Oliveira? Mas ele não escreveu nem uma única palavra desse poema. Nesse caso, se tem que pensar em uma autoria textual híbrida entre Camões e Antônio de Oliveira. Nós podemos imaginar que esses nomes (Camões e Antônio de Oliveira) seriam como campos de atração que vão, de algum modo, conformando a nossa leitura. Enfim, a criação muda, mas não podemos deixar de aprender com processos que vêm lá de trás. Não se deve cair na ilusão de que é possível construir um exercício de criação que seja totalmente novo, a partir do zero. Não é bem assim. Nunca foi, de fato!

\section{PALIMPSESTO}

5) Na sua visão, o contexto digital pode influenciar no ensino de Literatura no sentido de democratizar novas criações literárias possibilitadas pelo ambiente virtual?

\section{ALCKMAR LUIZ DOS SANTOS}

Indubitavelmente, em todos os sentidos. $\mathrm{O}$ acesso à literatura tradicional, impressa ou até mesmo a oral se ampliou enormemente. A democratização é evidente e a qualidade aumenta. Hoje, tenho acesso para as minhas aulas a materiais que são 
raríssimos e que se encontram em bibliotecas a que pouquíssimas pessoas teriam alcance. Eu, por exemplo, ponho em sala de aula, quando falo de século XVIII, algumas páginas de $O$ verdadeiro método de estudar (1746), do Verney, que é uma obra colossal, imensa, cujas reedições são raríssimas e caríssimas. Pego isso em uma edição digital que está disponível gratuitamente e discuto com os alunos. Portanto, a democratização, realmente, é muito ampla. Sem contar que as criações e literaturas digitais não são vendidas. Elas estão à disposição, há um interesse grande por elas, mas não existe um mercado grande de indivíduos que comprariam esse tipo de coisa. A relação com a fruição da obra de arte está mudando muito. Hoje em dia, as pessoas se dispõem a pagar muito pouco por um vídeo para assistir, uma música para ouvir, então acho muito difícil que alguém se disponha a pagar por uma criação literária digital. Devido a essas questões, estão, em geral, todas disponíveis e isso, evidentemente, democratiza também o acesso a elas.

\section{PALIMPSESTO}

6) A que o professor precisa estar atento quando se trata da leitura de textos literários na era da cultura digital?

\section{ALCKMAR LUIZ DOS SANTOS}

O professor precisa estar atento a muita coisa. Primeiramente, à confiabilidade da informação. Vou dar dois exemplos: fizemos uma edição de O Uraguai (1769), do Basílio da Gama, a partir de uma edição mais fácil e disponível. Pouco tempo depois, descobri, graças ao livro Mecenato Pombalino e Poesia Neoclássica (1999), do Ivan Teixeira, que havia notas do Basílio da Gama a esse poema. Fui atrás e refiz a edição digital. Só que nesse tempo, muita gente reproduziu o nosso arquivo sem as notas, já que a facilidade de reproduzir uma lacuna é muito grande. Com isso, as cópias em que faltavam as notas eram bem mais numerosas do que as que vinham completas.

Um segundo exemplo: Alberto Caeiro, heterônimo do Fernando Pessoa, tem dois versos que aparecem na edição da Ática, de Portugal, como: "Sinto-me nascido a cada momento / Para a eterna novidade do Mundo”. Só que no manuscrito original a palavra "eterna" está riscada e substituída por um garrancho que foi definido como 
“completa". Na edição da Ática ficou o "eterna". Se alguém procurar esse verso na internet, posso garantir que, na maioria dos casos, vai encontrar "eterna", que é o que Caeiro/Pessoa não queria que ficasse. O erro, portanto, se propaga.

Ao lado disso, temos, por exemplo, a Wikipédia. Muitos ainda têm preconceito com seu uso, porque no começo havia muita falha. Hoje em dia, continua havendo, mas ela é um auxiliar valiosíssimo no trabalho com a literatura ou com qualquer outra área. É necessário o cuidado de verificar o acerto da informação. Tanto o professor quanto o aluno precisam ter uma visão muito mais aguçada e crítica sobre o que estão consumindo para ver se é uma fonte fidedigna ou não.

\section{PALIMPSESTO}

7) No seu ponto de vista, as faculdades de Letras preparam os futuros professores de Literatura para ensinarem a disciplina em contextos digitais? Seria necessário repensar os currículos nesse sentido? Como?

\section{ALCKMAR LUIZ DOS SANTOS}

Talvez não seria necessário repensar propriamente os currículos, mas as práticas pedagógicas. Posso falar do meu caso no curso de Letras da Universidade Federal de Santa Catarina. Como disse anteriormente, poucos colegas ainda se dispõem a utilizar esse ferramental. Aqui, graças ao ensino a distância, contamos no ensino presencial com a plataforma Moodle, que é excelente. Desde 2008 uso essa plataforma, que é uma forma de trazer esses recursos do meio digital para a sala de aula. Eu dou aula com uma televisão grande ligada à internet e vou falando, mostrando e pesquisando. Entretanto, sei que poucos colegas fazem isso e que, em geral, nos cursos de Letras esse uso da tecnologia ainda está engatinhando.

Eu diria que reformular o currículo seria interessante, oferecendo uma disciplina sobre o uso de meios digitais no ensino, na criação e na leitura literária. Todavia, não é uma condição sine qua non, porque, se os professores utilizarem esses meios digitais e fizerem os alunos os usarem em todas as disciplinas ou na maioria delas, não é necessária uma disciplina específica. Isso já iria acontecer nas disciplinas tradicionais. 


\section{PALIMPSESTO}

8) Qual conselho você pode deixar para professores que ainda têm reservas quanto ao uso de tecnologias no ensino de Literatura?

\section{ALCKMAR LUIZ DOS SANTOS}

Eu diria que já usamos a tecnologia, afinal a escrita é uma técnica para registrar informação. O giz e o quadro negro também são tecnologias. Um smartphone que muitos ou quase todo mundo usa é uma tecnologia. A questão, portanto, é não demonizar a tecnologia. A tecnologia, a priori, não é boa, nem má. Ela vai ser boa ou má, sempre dependendo do uso que damos a ela. A radioatividade que faz tomografia e salva vidas é a mesma que matou milhares de pessoas no Japão, na Segunda Guerra Mundial. O grande ponto, então, é o uso que fazemos disso. A internet pode ser uma arma de liberação democrática sim, mas pode ser uma terrível arma de controle social e de manipulação. Vimos a eleição de um psicopata aqui como presidente graças, em boa parte, a manipulação de redes sociais. Se nós nos omitirmos, outros vão usar e vão dar o sentido que querem a elas. Eu sempre brinco e digo que nós, das Humanas, temos que botar a mão na massa e não deixar o meio digital para engenheiros e cientistas da computação. Temos que civilizá-los e direcionar o uso das tecnologias para a nossa visão humanística. Isso é essencial. Se deixarmos só com os técnicos, a maioria deles vai fazer um uso muito pragmático e bastante comercial. Felizmente, não são todos assim. O pessoal da computação com quem trabalho é maravilhoso. Eles têm uma concepção extremamente aberta da computação e do benefício que pode trazer à sociedade. Tenho sorte de trabalhar pessoas que têm essa visão abertíssima como a deles, mas muitos não são assim. Então eu acho que é um dever nosso botar a mão na tecnologia digital e usá-la para dar um sentido humanístico a ela.

\section{Referências}

SANTOS, Alckmar Luiz dos. Rios Imprestáveis. São Paulo: Lemos Editorial, 2001. 
SANTOS, Alckmar Luiz. Ao que minha vida veio... 1. ed. Florianópolis: Editora UFSC, 2011. 


\title{
Technological Metamorphism in the Classroom: an Interview with Professor Alckmar Luiz dos Santos
}

\author{
Alckmar Luiz dos Santos ${ }^{\text {iv }}$ \\ Interviewers: \\ Gabriela Ribeiro Nunes ${ }^{v}$ \\ Marcela Santos Brigida ${ }^{\text {vi }}$
}

Translated by

Gabriela Ribeiro Nunes

Marcela Santos Brigida

One of the interviewees in this edition of Palimpsesto is Alckmar Luiz dos Santos, a professor at Universidade Federal de Santa Catarina. With a BA in Electronic Engineering from Universidade Estadual de Campinas, he holds an MA in Literary Theory and History (UNICAMP) and a PhD in Literary Studies from the Université Paris VII. Since 1995, he has acted as a coordinator of Núcleo de Pesquisas em Informática, Literatura e Linguística (NUPILL), where he develops research on literary texts in digital media, exploring the resources that information technology and communication technologies bring to research in Literature and Linguistics. He is also a poet, novelist, essayist, and digital creator. Among his vast literary production, we would highlight the poetry book Rios Imprestáveis (2001), winner of the Redescoberta da Literatura Brasileira da Revista Cult Award and the novel Ao que minha vida veio... (2011), winner of the Romance Salim Miguel Award.

\footnotetext{
iv Alckmar Luiz dos Santos has a degree in Electronic Engineering from Universidade Estadual de Campinas (1983), a master's degree in Theory and Literary History from Universidade Estadual de Campinas (1989) and a PhD in Literary Studies from the Université Paris VII (1993). Since 1994, he has been a professor of Brazilian Literature at Universidade Federal de Santa Catarina (UFSC) and, since 1995, a coordinator at Núcleo de Pesquisas em Informática, Literatura e Linguística (NUPILL). He was a guest researcher at Université Paris 3 - Sorbonne Nouvelle (2000-2001) and at Universidad Complutense de Madrid (2009-2010). | NUPILL website: http://nupill.ufsc.br/

${ }^{v} \mathrm{PhD}$ student in Literary Theory/Comparative Literature at Universidade do Estado do Rio de Janeiro (UERJ). https://orcid.org/0000-0002-6593-0351 |j15c17g11@ hotmail.com

${ }^{\text {vi }} \mathrm{PhD}$ Student in Literary Studies at Universidade do Estado do Rio de Janeiro (UERJ). CNPq Scholar. https://orcid.org/0000-0002-0951-1603 | marcela.brigida@uerj.br
} 
Professor Alckmar's outstanding curriculum, while a reflection of his prominence as a researcher, teacher, and writer, did not allow us to anticipate how kind and humble he would reveal himself to be throughout this interview. We are immensely grateful for the considerate way in which he accepted our invitation to talk to us for this edition, that brings "Literature Teaching in Digital Contexts: Dialogues and Connections" as its theme. It was a rewarding and enriching experience to learn through the reflections he proposed regarding the use of digital technologies in the classroom, besides the recommendations of tools that might aid professors in the context of literature teaching. His modesty in citing his colleagues - as well as the didactical way in which he conveyed his ideas - inspire and invite us to update our teaching practices following in his footsteps.

\section{PALIMPSESTO}

1) During a pandemic, more than ever, teaching and learning in a digital context has been a reality for teachers and students. As a researcher in that field, what are the advantages and disadvantages that such an environment brings when it comes to literature teaching?

\section{ALCKMAR LUIZ DOS SANTOS}

As a professor, I have had some experiences with distance learning that have not always been positive. Firstly, because there was some pressure from the Ministry of Education to transform distance learning into numbers to be advertised, with no concern towards quality. When we were preparing the first distance undergraduate course in Letras, at UAB, Universidade Aberta do Brasil, a Ministry of Education representative came to talk to us, to lay down the guidelines. He said that students would have five to ten hours a week of study. That was what we had to rely on. They would receive the same degree as the others who would have devoted between 30 and 40 hours a week, which suggests that they would all acquire the same level of education. Evidently, this does not add up.

On the other hand, there were students who thought that distance learning was synonymous with laxity, that it would require less effort to fulfil their degrees. 
However, the necessary work is much ampler, as the students need more discipline, awareness, attention and understanding of what they can do and of what they are going to do. They must also extract as much as possible from the platforms, the material available, the professors, the tutors. Therefore, there are problems that must be considered from all sides: from the institutions that organize this form of teaching, from professors, and from students. During the pandemic, this process has been accelerated, but, in principle, I do not see this turn towards remote education as necessarily or exclusively a bad thing. There was much fear from my colleagues, professors, of using this type of resource, when, in fact, there should be no fear. We must use it and improve it as much as we can. If problems are due to appear, we will only be able to solve them through our experiences. The pandemic, therefore, has helped in this regard, since now everyone has become involved with it.

\section{PALIMPSESTO}

2) You have been a coordinator of Núcleo de Pesquisas em Informática, Literatura e Linguística [Center for Research in Informatics, Literature and Linguistics] (NUPILL), since 1995. Where did your interest in thinking about the relationship between Informatics and Literature come from and what is the importance of understanding this dynamic for a literature professor? In what sense did this influence your teaching practices?

\section{ALCKMAR LUIZ DOS SANTOS}

When I joined the Universidade Federal de Santa Catarina, I had recently finished my PhD. I worked with epistemology, literary criticism, philosophy, poetry, literature. However, my first degree was in Electronic Engineering. I cannot say today that I am an electronic engineer, not even by far. Those are things that have remained in a remote and indistinguishable past. Now, I remember quite little, but the reasoning basis has remained with me, as well as an interest and a certain ease in using digital tools.

From the final months of my MA at UNICAMP, I was already using a text editor for the elaboration of my dissertation. After that, during my $\mathrm{PhD}, \mathrm{I}$ used the 
computer a lot. I also used a database to catalog my readings, besides searching the contents of those readings, since many of the reports I made were digitized. Consequently, I was already familiar with the technology. Besides, my creative relationship with literature started when I was 8; it was in that age that I decided to be a poet. This entire trajectory made me, some time later, start to ask myself why I was not using digital tools for literary creation, since I used it for my academic production. That was how the first flash came. Alongside this, there was also the pragmatic side of testing to see what would happen. I started developing creative experiments with my friend Gilbertto Prado, a professor at USP.

Reflecting on the use I made of digital tools for the production of my thesis, as well as of essays and articles, I came to the conclusion that it would be interesting to have access to documents, resources and information in the literary field in a quick way, using the internet. Thus, the idea of creating a digital library came, starting with Machado de Assis's oeuvre. This was the initial objective of NUPILL, in 1995. Soon other goals were added to this, such as the idea of creating a database of literary history associated with the digital library. This idea was not mine. I got it from the Hubert de Phalèse group, from Paris 3, where I did my postdoctoral research, between 2000-2001. To this day, the French literature database remains available. In our case, we built a Brazilian literature database.

All of this worked very well in my teaching practices. I'm just sorry that many colleagues don't use it as well. The level of convenience we have today to access and cross-reference data and information is enormous, and the digital library database is already quite large and continues to grow. Aside from the fact that I am one of those who helped to build all of this, the digital library and the database are invaluable assets for any literature professor. We have around 80 thousand literary works registered and around 6,500 digitalized works. We have more than 22 thousand authors of Portugueselanguage literature - from Brazil, Portugal and Africa. From Africa, unfortunately, there are still very few; Portugal's numbers are increasing now; the bulk of our data comes from Brazil. This is quite helpful, as it allows access to the works, information about authors, pseudonyms, subheadings, genres. While teaching, I constantly say: "Let's check this in the digital library, let's verify this in the database". If anyone wants to know, for example, how many theatre books were published between 1850-1880 by 
women in Portugal, this is something that is easily found in our database. Not to mention that, little by little, thanks to the work with a friend and computer researcher at UFSC, Roberto Willrich, we developed tools such as DLNotes, for annotations in literary files and HTML files. In our case, we use it for literary works. This annotation tool is quite useful in the classroom since it streamlines and enriches the students' reading. After we started using DLNotes in 2010, the classes, the dialogue with students and what they bring as information and understanding of literary works have improved a lot.

There is also another friend, Adiel Mitmann (and also a researcher at NuPILL), who developed a tool throughout his $\mathrm{PhD}$ that is called Aoidos. It is responsible for automatically scanning verses, regardless of size. It will scan both a quatrain, four verses, in milliseconds, and a corpus of a million verses, quantifying all the syllabic accommodation phenomena, such as synaloepha, diaeresis, synaeresis, ectlipse, suarabácti etc. Moreover, it is capable of mapping and quantifying the rhythms and meters used by poets, being, therefore, an extremely valuable tool for those who work with verses. The point now is that our fellow professors need to be willing to take the step of diving into digital technology. If we use both cellphones and smartphones, to employ those digital tools is not so much of an effort.

\section{PALIMPSESTO}

3) What major changes have you seen, as NUPILL's coordinator for over 25 years, in the way we deal with literary texts and in the process of teaching/learning literature in a digital context?

\section{ALCKMAR LUIZ DOS SANTOS}

In regard to teaching/learning literature, it is basically what I answered in the previous question. I have observed that my colleagues are increasingly interested in it, especially the younger ones who are arriving at universities. They are already familiarized with all of this, so their comfort while using these tools and strategies is much bigger and that makes a lot of difference. 
When it comes to dealing with literary texts, in the 1990s and 2000s, there was great amount of euphoria around that. In the United States, there are names like Michael Joyce and there is my friend in France, Philippe Bootz, who are working on this. Many people, including me, were also looking for new paths. In my case, I have always sought to make new elements establish a dialogue with old ones. I have never believed in the construct of starting a creation or a thought from scratch. This reminds me of that beautiful literary work that is Ecclesiastes (1:9): "There is nothing new under the sun". The idea of what is new is often a new way of using what came before and that you have learned from others.

There was, then, a very intense search on one side and on the other, with varying results, but I would say that they were all interesting. With no intention of being indulgent, it is a beautiful attempt that is still being made today to seek to establish a dialogue with the new and with these new means. Those are new dynamics, new spaces and a new way of thinking about literary questions. In fact, what we saw was an attempt, sometimes disguised, sometimes explicit, to reconcile three axes. One axis pertains to verbal literature, the other one is semiotic, that is, the image and sound that gain new relevance in these creations, and the last one is the computational one. The three axes (verbal, semiotic and computational) always appear. The authors and creators that I find most interesting were aware of this and tried to make these three axes establish a dialogue in an interesting and enriching way, each with its own style. Others did this without much awareness, so we saw many cases of electronic poetry or digital poetry which, in fact, was the old verse that was made for the print tradition, which was simply placed on a computer screen; just as many people spoke of digital literature, computerized literature, computational literature, electronic literature, when it was simply literature made on paper, digitized and made available to read on the screen. Few people did what would be true digital or computational or electronic literature. In my case, I would always rather call digital literatures those that cannot be printed, because the role of computational interaction is too significant. The interaction between the various semiotics with the verbal element is very intense as well.

After this euphoria of learning, what I perceive today is that there are some strands that are already consolidating. One of them is Natural-language generation, which has been going on since the 1950s; there are still a lot of people working with it 
in an interesting way. Another aspect that has been greatly strengthened in recent years is the dialogue with video games. In fact, a literary theorist, Espen Aarseth, said that he stopped being a literary critic to become a student of the games. There are those who work more with the visual side. In our case, for example, the last creation we made, and we are ending now is called e-Imigrações. It is about reconciling verbal creation with the semiotic data of sound and image and with computational interactivity, bringing the visual side of the story in a comic. Therefore, there is a very heterogeneous range of elements that can be brought together to give them meaning. The problem is this more univocal sense, because it is often easy to join disparate and heterogeneous elements and, in the end, to come across a Frankenstein-like construction that does not move, is dead and does not walk - unintelligible parts artificially associated.

\section{PALIMPSESTO}

4) How do you think online contexts influence the way of producing and consuming literature? Is there a reconfiguration of the role of the author, the reader, and the literary text itself?

\section{ALCKMAR LUIZ DOS SANTOS}

Of course. To begin with, in terms of literary theory, a very clear difference must be made between author and writer. The writer is the person made of flesh, bone, blood, life, who has a tax-payer's registry number, as well as a family, a profession, weight, height, eye color, hair. The author, on the other hand, is a textual element that will appear when there is a reading - understood in the widest possible way: from reading words on a printed sheet of paper to reading an interactive digital work. The author, then, is a textual element that is part of the reading process.

Regarding the new configurations, there is, for example, collective processes of writing and creation. When a creation like the one I referred to in the previous question, e-Immigration, is produced, I cannot claim that I am the creator. The creators are Vinícius Rutes, Rafael Duarte and me. The three of us are working on it, each with their own contribution. Evidently, I move more to the side of verbal creation and interactions, 
which is something I have been dealing with for a long time. Rafael leans more towards visuality. His thesis was about graphic novels and visual poetry. Vinícius, on the other hand, has collaborated a lot with the programming, which Rafael and I have not mastered, without leaving aside an awareness of the verbal aspect. Each is responsible for a specific part but participates in the others as well. Thus, it is a collective creation. However, these different creations by different authors are not specific to digital culture. The centos - a type of poetic composition from the Middle Ages, in which medieval poets took verses from Virgil's Aeneid, combined them and made a poem - are an example of that. This tradition has been perpetuated. In the eighteenth century, at the Brazilian Academies, a poet called Antônio de Oliveira produced a poem called: "Soneto encontrado no poema do príncipe dos poetas espanhóis" [Sonnet found in the poem of the prince of Spanish poets]. The prince of Spanish poets is Camões. At the time, Spain was the Iberian Peninsula. "Spanish" was understood as we now understand “Iberian”. Antônio de Oliveira took fourteen lines from Os Lusíadas and made a sonnet to honor the Academy of which he was a member. The poem is syntactically coherent, semantically correct, it is a sonnet, with heroic decasyllable verses, the rhyme scheme is the most traditional, but... who is the creator of this sonnet? Camões? The verses are his, but Camões never imagined that this sonnet would be within Os Lusíadas. So, is the creator Antônio de Oliveira? He did not write a single word of that poem. In this case, one must think of a hybrid textual authorship between Camões and Antônio de Oliveira. We can imagine that those names (Camões and Antônio de Oliveira) would be like attraction fields that, in some way, conform our reading. Ultimately, creation changes, but we cannot help but learn from processes that come from the past. One should not fall into the illusion that it is possible to build a creative exercise that is completely new, from scratch. It is not so. It never has been, in fact!

\section{PALIMPSESTO}

5) From your your point of view, could the digital context influence Literature teaching in the sense of democratizing new literary creations made possible by a virtual environment? 


\section{ALCKMAR LUIZ DOS SANTOS}

Undoubtedly, in every way. The access to traditional literature, in print or even oral form, has expanded enormously. Democratization is evident and quality increases. Now, I have access in my classes to materials that are very rare and that are found in libraries that very few people would have access to. I, for example, will display in the classroom, when I speak about the eighteenth century, a few pages of Verney's $O$ verdadeiro método de estudar [The True Method of Studying] (1746), which is a colossal, immense work, whose reissues are very rare and very expensive. I use a digital edition that is available for free and discuss it with the students. Therefore, the process of democratization is really quite far-reaching. Not to mention that digital creations and literature are not actually sold. They are available, there is a great interest in them, but there is not a large market for individuals who would buy this type of thing. The relationship with the enjoyment of the work of art is changing a lot. Today, people are willing to pay very little to watch a video, to listen to a song, so I find it very difficult for anyone to be willing to pay for a digital literary creation. Due to such issues, they are, in general, all freely available and that, of course, also democratizes the access to them.

\section{PALIMPSESTO}

6) What does a professor need to be aware of when it comes to reading literary texts in the era of digital culture?

\section{ALCKMAR LUIZ DOS SANTOS}

A professor needs to be aware of many things. First, the reliability of the information. I will give you two examples: we made an edition of O Uraguai (1769), by Basílio da Gama, from a more easily accessible edition. Shortly thereafter, I discovered, thanks to the book Mecenato Pombalino e Poesia Neoclássica (1999), by Ivan Teixeira, that there were notes from Basílio da Gama to this poem. I went back and redid the digital edition. The problem is that, throughout that time, many people reproduced our 
file without the notes, as it quite easy to reproduce a gap. Ultimately, there were many more copies in which the notes were missing than the ones that were complete.

A second example: Alberto Caeiro, Fernando Pessoa's heteronym, has two verses that are included in Ática's edition, from Portugal, as: "Sinto-me nascido a cada momento / Para a eterna novidade do Mundo" ["I feel at every moment that I am being born / Into the eternal novelty of the World"]. However, in the original manuscript the word "eterna" ["eternal"] was crossed out and replaced by a scribble that has been read as "completa" ["complete"]. In Ática's edition we find the word "eterna". If a person looks up that verse online, I can guarantee that, in most cases, they will find the "eterna" variant, which Caeiro/Pessoa had crossed out. The error, therefore, keeps on being propagated.

Besides that, we have, for example, Wikipedia. Many people are still prejudiced against its use, because in the beginning there were many flaws in it. Those still exist now, but it is a highly valuable assistant while working with literature or any other area. One must be cautious to verify the accuracy of the information. Both professors and students must have a much sharper and critical view of what they are consuming to check if it is a reliable source or not.

\section{PALIMPSESTO}

7) In your view, do Letras institutes prepare future literature professors to teach their courses in digital contexts? Would it be necessary to rethink curricula in that regard? How?

\section{ALCKMAR LUIZ DOS SANTOS}

Perhaps it would not be necessary to rethink curricula, but pedagogical practices. I can talk about my experience in the Letras course at the Universidade Federal de Santa Catarina. As I have said earlier, very few colleagues are willing to use that tool yet. Here, due to distance learning, we use the Moodle platform (which is excellent) for face-to-face teaching. I have been using this platform since 2008. It is a way to bring those resources from the digital environment to the classroom. I teach with a largescreen TV connected to the internet and I engage in speaking, presenting and 
researching. However, I know that few colleagues do this and, in general, in Letras courses the use of technology is still in its baby steps.

I would say that reformulating the curriculum would be interesting, offering a course on the use of digital media in teaching, creation, and literary reading. However, it is not a sine qua non condition, because if professors use digital media and make students use them in all subjects or in most subjects, a specific subject would not necessary. This would already happen in traditional courses.

\section{PALIMPSESTO}

8) What advice would you give to professors who still have reservations about the use of technologies in literature classes?

\section{ALCKMAR LUIZ DOS SANTOS}

I would say that we already use technology, after all writing is a technique for recording information. Chalk and the blackboard are also technologies. A smartphone that many people, or almost everyone, use is a piece of technology. The question, therefore, is not to demonize technology. Technology, a priori, is neither good nor bad. It will be good or bad, always depending on the attribution we give to it. The radioactivity that is used in tomographies and saves lives is the same that killed thousands of people in Japan, in the Second World War. The point, then, is what we make of it. The internet can be a weapon of democratic liberation, but it can be a terrible weapon of social control and manipulation. We saw the election of a psychopath as president in Brazil thanks, in large part, to the manipulation of social networks. If we omit ourselves, others will use it and give it the meaning they want. I always joke and say that we, from the Humanities, have to engage in a hands-on approach and not leave the digital medium to engineers and computer scientists. We must civilize it and direct the use of technologies to our humanistic vision. This is essential. If we leave it to the technicians, most of them will use it very pragmatically and commercially. Fortunately, not everyone is like that. The computer people I work with are wonderful. They have an extremely open conception of computing and the benefit it can bring to society. I am lucky to work with people who have an open view like theirs, but many are not that 
way. Finally, I think it is our duty to get involved with digital technology and use it to give it a humanistic meaning.

\section{References}

SANTOS, Alckmar Luiz dos. Rios Imprestáveis. São Paulo: Lemos Editorial, 2001.

SANTOS, Alckmar Luiz. Ao que minha vida veio... 1. ed. Florianópolis: Editora UFSC, 2011. 